\title{
On the Crystalline Structure, Stoichiometry and Band Gap of InN Thin Films
}

K. M. Yu ${ }^{\text {a) }}$, Z. Liliental-Weber ${ }^{\text {a) }}$, W. Walukiewicz ${ }^{\text {a) }}$, S. X. Li ${ }^{\text {a),b) }}$, R. E. Jones ${ }^{\text {a),b) }}$, W. Shan $^{\text {a) }}$, J. W. Ager III ${ }^{\text {a) }}$, E. E. Haller ${ }^{\text {a),b) }}$, Hai Lu ${ }^{\text {c)}}$, and William J. Schaff ${ }^{\text {c) }}$

a) Materials Sciences Division, Lawrence Berkeley National Laboratory, Berkeley, California 94720

b) Department of Materials Science and Engineering, University of California, Berkeley, California 94720

${ }^{c)}$ Department of Electrical and Computer Engineering, Cornell University, Ithaca, New York 14853

\section{ABSTRACT}

Detailed transmission electron microscopy (TEM), x-ray diffraction (XRD), and optical characterization of a variety of InN thin films grown by molecular beam epitaxy under both optimized and non-optimized conditions is reported. Optical characterization by absorption and photoluminescence confirms that the band gap of single crystalline and polycrystalline wurtzite $\mathrm{InN}$ is $0.70 \pm 0.05 \mathrm{eV}$. Films grown under optimized conditions with a AlN nucleation layer and a GaN buffer layer are stoichiometric, single crystalline wurtzite structure with dislocation densities not exceeding mid- $10^{10} \mathrm{~cm}^{-2}$. Non-optimal films can be poly-crystalline and display an XRD diffraction feature at $2 \theta \approx 33^{\circ}$; this feature has been attributed by others to the presence of metallic In clusters. Careful indexing of wide angle XRD scans and selected area diffraction patterns shows that this peak is in fact due to the presence of polycrystalline InN grains; no evidence of metallic In clusters was found in any of the studied samples. 
InN has been the least studied of the group III-nitride semiconductors. Early optical absorption studies [1] on sputtered InN films with relatively high electron concentrations $\left(>10^{19} / \mathrm{cm}^{3}\right)$ and low mobilities $\left(<100 \mathrm{~cm}^{2} / \mathrm{V} \cdot \mathrm{s}\right)$ were used to derive a fundamental bandgap of $\sim 1.9 \mathrm{eV}$. For over a decade it had been accepted that the band gap of InN was $1.9 \mathrm{eV}$. However, recent measurements on high quality InN films with low electron concentrations $\left(<10^{19} / \mathrm{cm}^{3}\right)$ and high mobilities $\left(>100 \mathrm{~cm}^{2} / \mathrm{V} \cdot \mathrm{s}\right)$ grown by molecular beam epitaxy (MBE) and metalorganic vapor phase epitaxy (MOCVD) have unambiguously agreed upon a fundamental band gap of $0.70 \pm 0.05 \mathrm{eV}[2-4]$. This new small gap for InN was further supported by recent ultrafast differential transmission measurements performed on MBE-grown material [5]. Studies of InN samples with free electron concentrations spanning three orders of magnitude, up to $10^{21} \mathrm{~cm}^{-3}$, have revealed a large Burstein-Moss shift in the observed optical absorption edge from $0.7 \mathrm{eV}$ to $1.7 \mathrm{eV}$ [6]. This effect provides an explanation for the wide range of $\mathrm{InN}$ band gaps reported previously. With this new band gap value for $\mathrm{InN}$, it is now established that the fundamental direct band gap range of the III-nitride alloy system is the widest of any compound semiconductor, extending from InN (0.7 eV, near-IR), to GaN (3.4, mid-UV), and finally to AlN (6.2 eV, deep-UV) [7].

The interpretation of optical absorption and photoluminescence (PL) data from InN has been questioned recently by Shubina et al. [8]. These authors claimed that the absorption edge of $\mathrm{InN}$ at $0.7 \mathrm{eV}$ actually results from resonant Mie scattering by metallic indium clusters and that $\mathrm{PL}$ at $\sim 0.7 \mathrm{eV}$ in $\mathrm{InN}$ originates from optical transitions involving interface states between the In clusters and the InN matrix [8]. They assigned an “anomalous" peak from x-ray diffraction spectra at $2 \theta \sim 33^{\circ}$ to metallic In. Large 
deviations from stoichiometry in $\mathrm{InN}$ films grown by various methods have been reported [8,9]. Shubina et al. concluded that the observed band gap variations (from 0.7-1.9 eV) in InN originate from large deviations from stoichiometry due to different film growth conditions $[8,10]$. To address the issue of the relationship between the structural and optoelectronic properties of $\mathrm{InN}$ we have applied detailed structural and optical probes to investigate MBE samples grown under different conditions. Here we present results of $\mathrm{x}$ ray diffraction (XRD), transmission electron microscopy (TEM), and Rutherford backscattering spectrometry (RBS) along with optical and electrical characterization to demonstrate that high quality, stoichiometric $\mathrm{InN}$ has an optical gap of $\sim 0.7 \mathrm{eV}$. We find that the "anomalous" diffraction peak assigned to metallic In by Shubina et al. is due to the presence of polycrystalline $\mathrm{InN}$ grains in films grown under non-optimal growth conditions.

InN films were grown on sapphire substrates by molecular beam epitaxy $[11,12]$. The optimized growth method that results in epitaxial InN films with low electron concentration, high mobility and low dislocation density, uses an AIN nucleation layer and a GaN buffer layer deposited prior to InN growth. Non-optimized films grown directly on sapphire without the nucleation and buffer layers were also studied. InN film thicknesses ranged from $80 \mathrm{~nm}$ to over $2 \mu \mathrm{m}$ for the samples used in this study. The films used here were nominally undoped with electron concentrations measured by Hall effect ranging from high $10^{17} \mathrm{~cm}^{-3}$ to mid- $10^{19} \mathrm{~cm}^{-3}$. InN films with relatively low electron concentrations had room temperature mobilities in excess of $1000 \mathrm{~cm}^{2} \mathrm{~V}^{-1} \mathrm{~s}^{-1}$.

XRD in the theta-two-theta coupled geometry using $\mathrm{Cu} \mathrm{K}_{\alpha} \mathrm{X}$-rays was employed to evaluate the presence of secondary phases and/or polycrystallinity. The microstructure 
of the InN films was investigated in detail by TEM in cross-section and plan-view configurations. A JEOL 3010 transmission microscope operating at $300 \mathrm{keV}$ was used in classical bright field, high resolution, and selected area electron diffraction (SAD) modes. Care was taken in the sample preparation and examination to avoid conditions that would decompose the InN and potentially form metallic In.

Optical absorption measurements were performed with a CARY-2390 NIR-VIS-

UV spectrophotometer. The direct bandgap was determined by the standard procedure of extrapolating the linear part of the squared absorption coefficient. Photoluminescence (PL) measurements were performed at room temperature in the back-scattering geometry by excitation at $515.4 \mathrm{~nm}$. The signals were then dispersed by a SPEX 1680B monochromator and detected by either a liquid-nitrogen cooled InSb photodiode or a photomultiplier, as appropriate.

Rutherford backscattering spectrometry (RBS) measurements reveal that all of the studied films are stoichiometric to within the measurement error of $\pm 3 \%$. Figure 1 (a) shows the XRD pattern of a typical optimized InN film in which only the (0001) diffraction peaks of wurzite InN, GaN (buffer layer), AlN (nucleation layer), and sapphire (substrate) are observed. The inset in this figure shows a high resolution scan in the region of the InN 0002 diffraction $\left(30^{\circ}<2 \theta<37^{\circ}\right)$; no peak in the vicinity of $33^{\circ}$ is observed. The high structural quality of InN films grown on $\mathrm{GaN}$ buffer layers is confirmed by the transmission electron micrograph shown in Fig. 2 (a). For all of the optimized MBE-grown films studied the measured dislocation densities do not exceed mid- $10^{10} \mathrm{~cm}^{-2}$. As a comparison, GaN films grown on sapphire by MBE typically have $10^{9}$ dislocations $\mathrm{cm}^{-2}$. Most of the dislocations were of edge character. Selected area 
electron diffraction (SAD) patterns (shown in the inset of Fig. 2 (a)) consist of a single wurtzite InN diffraction pattern with no additional spots which could suggest the existence of secondary phases or metallic In.

All the samples with the structural characteristics of good quality InN show both strong room temperature PL and a well resolved absorption edge at about $0.7 \mathrm{eV}$. Figure 3 shows the PL and absorption spectra (dashed) from the sample shown in Fig. 2(a). The absorption curve for this sample is smooth and does not show signs of any additional edges and/or resonances.

Our XRD results are in sharp contrast to the results presented in reference 8 that showed a diffraction peak at $2 \theta \approx 33^{\circ}$ with intensity up to a third of that from the (0002) diffraction peak of the host InN film. The authors attribute this "anomalous" diffraction peak to metallic In present in "non-stoichiometric In-rich" InN film. We note that such a pronounced (101) diffraction peak of metallic In could be observed only if there were a large density of large, micron-sized In clusters present in the films.

To elucidate the origin of the "anomalous" $33^{\circ} \mathrm{XRD}$ peak we have investigated a InN film grown directly on sapphire without the AlN nucleation layer and the GaN buffer layer. As a result of these non-optimal MBE growth conditions, this $\operatorname{InN}$ film is expected to be of poor crystalline quality. RBS measurement aligned in the growth direction shows that the film is a $80 \mathrm{~nm}$ thick stoichiometric $\mathrm{InN}$ with poor epitaxial quality (minimum yield channeling yield $\chi \sim 0.90$ ). The XRD pattern for this film (GS1489) is shown in Fig. 1 (b); a peak at $33^{\circ}$ is observed. The XRD pattern shown in Fig. 1 (b) also shows that in addition to the $33^{\circ}$ peak, diffraction peaks at $\approx 52^{\circ}(\mathrm{d}=1.76 \AA), 63^{\circ}$ 
$(\mathrm{d}=1.48 \AA)$, and $69^{\circ}(\mathrm{d}=1.36 \AA)$ are also present. These, and the $33^{\circ}$ peak, can all be assigned to diffraction peaks of random InN grains.

Additional evidence for the absence of metallic In in this film was obtained by TEM. A typical micrograph and electron diffraction pattern of the InN film grown directly on sapphire (GS1489) are shown in Fig. 2(b). The micrograph and the ring pattern in the SAD (inset) clearly reveal that the film is polycrystalline in nature. Using the sapphire substrate as an internal standard to precisely determine the camera length, all consecutive diffraction rings in the SAD pattern could be assigned to InN. The interplanar distances $d$ obtained from the SAD and XRD from GS1489 are tabulated in Table I. Our experimental $d$ values (TEM and XRD) are in excellent agreement with calculated values for wurzite $\mathrm{InN}$.

We note that the $\operatorname{InN} \mathrm{d}_{(1011)}$ of $2.69 \AA$ is very close to the $\mathrm{d}_{101}$ of metallic In (2.72 $\AA$ ). This $d$ spacing gives rise to a diffraction peak at $33^{\circ}$ in XRD. However, none of the other interplanar distances which would be expected from In such as $002(2.47 \AA)$, $110(2.3 \AA)$ or $112(1.68 \AA)$ were observed in either the SAD or XRD pattern. Some larger grains were studied individually by SAD and patterns consistent with $\mathrm{InN}$ in different orientations were observed. The RBS, XRD, and TEM results from the InN film GS1489 grown directly on sapphire show that the film is polycrystalline and stoichiometric. In this film, the "anomalous" $33^{\circ}$ peak is in fact the (011) diffraction peak of InN. No evidence of metallic In is observed. Thus we consider it likely that the $33^{\circ} \mathrm{XRD}$ line observed in some InN films and assigned to In in the literature $[8,10]$ is due to polycrystalline InN and not to metallic In. It should be noted that the 
polycrystalline InN films showed both PL peaks and the absorption edges at energies similar to those found in single crystal film (solid line in Fig. 3).

In conclusion we have studied the stoichiometry, structure and band gap of a variety of InN thin films grown by MBE using Rutherford backscattering, X-ray diffraction, transmission electron microscopy, photoluminescence, and optical absorption. We found that InN films grown by MBE on a GaN buffer layer are stoichiometric, single crystalline wurtzite structure with dislocation densities not exceeding mid- $10^{10} \mathrm{~cm}^{-2}$. The previously reported [8] "anomalous" diffraction peak observed in lower quality films and attributed to metallic In clusters is in fact due to the presence of polycrystalline InN grains in samples grown under non-optimized growth conditions. The band gap of stoichiometric, single and poly-crystalline wurtzite $\mathrm{InN}$ is measured to be $0.7 \mathrm{eV}$.

This work is supported by the Director, Office of Science, Office of Basic Energy Sciences, Division of Materials Sciences and Engineering, of the U.S. Department of Energy under Contract No. DE-AC03-76SF00098. The work at Cornell University is supported by ONR under Contract No. N000149910936. 


\section{REFERENCES}

1. T. L. Tansley and C. P. Foley, J. Appl. Phys., 59, 3241 (1986).

2. V. Yu. Davydov, A. A. Klochikhin, R. P. Seisyan, V. V. Emtsev, S. V. Ivanov, F. Bechstedt, J. Furthmüller, H. Harima, A. V. Murdryi, J. Aderhold, O. Semchinova, and J. Graul, Phys. Stat. Sol. B 229, R1 (2002).

3. J. Wu, W. Walukiewicz, K. M. Yu, J. W. Ager III, E. E. Haller, H. Lu, W. J. Schaff, Y. Saito, and Y. Nanishi, Appl. Phys. Lett. 80, 3967 (2002).

4. K. Sugita, H. Takatsuka, A. Hashimoto, and A. Yamamoto Phys. Stat. Sol. (b) 240, 421 (2003).

5. Fei Chen, A. N. Cartwright, Hai Lu, William J. Schaff, J. Cryst. Growth 269, 10 (2004).

6. J. Wu, W. Walukiewicz, W. Shan, K.M. Yu, J.W. Ager III, E. E. Haller, Hai Lu and William J. Schaff, Phys. Rev. B66, 201403 (2002).

7. J. Wu, W. Walukiewicz, W. Shan, K.M. Yu, J.W. Ager III, S. X. Li, E.E. Haller, Hai Lu, and William J. Schaff, Solid State Com. 127, 411 (2003).

8. T. V. Shubina, S. V. Ivanov, V. N. Jmerik, D. D. Solnyshkov, V. A. Vekshin, P. S. Kop'ev, A. Vasson, J. Leymarie, A. Kavokin, H. Amano, K. Shimono, A. Kasic, and B. Monemar, Phys. Rev. Lett. 92, 117407 (2004).

9. K. S. A. Butcher, M. Wintrebert-Fouquet, P. P.-T. Chen, T. L. Tansley, H. Dou, S. K. Shrestha, H. Timmers, M. Kuball, K. E. Prince, and J. E. Bradby, J. Appl. Phys. 95, 6124 (2004).

10. T. V. Shubina, S. V. Ivanov, V. N. Jmerik, M. M. Glazov, A. P. Kalvarskii, M. G. Tkachman, A. Vasson, J. Leymarie, A. Kavokin, H. Amano, I. Akasaki, 
K. S. A. Butcher, Q. Guo, H. Lu, W. J. Schaff, B. Monemar, and P. S. Kop'ev, Phys. Stat. Sol. (c), in press (2004).

11. H. Lu, William J. Schaff, Jeonghyun Hwang, Hong Wu, Wesley Yeo, Amit Pharkya, and Lester F. Eastman, Appl. Phys. Lett. 77 2548-2550 (2000).

12. H. Lu, Schaff, W.J.; Jeonghyun Hwang; Hong Wu; Koley, G.; Eastman, L.F. Source, Appl. Phys. Lett. 79 1489-91 (2001). 


\section{FIGURE CAPTIONS}

Fig. 1 X-ray diffraction patterns from (a) a typical InN film grown on a GaN/AlN buffer layers on sapphire. The inset in this figure shows a high resolution scan in the region of the 0002 diffraction $\left(2 \theta\right.$ of $\left.30-37^{\circ}\right)$. (b) XRD pattern from a InN film grown directly on sapphire (GS1489). A high resolution scan around the $33^{\circ}$ peak is also shown in the inset.

Fig. 2 (a) Cross-section TEM micrograph of a good quality InN film. The inset shows monocrystalline diffraction pattern of InN with 1010 zone axis. (b) Plan-view TEM micrograph of a polycrystalline $\mathrm{InN}$ film, the inset shows the characteristic ring pattern of the SAD data. The interplanar distances obtained from indexing the rings are in Table 1.

Fig. 3 Photoluminescence (PL) and absorption spectra of (a) a good quality single crystalline InN film grown with a AlN nucleation layer and a GaN buffer layer (dashed line) and (b) a polycrystalline InN film grown directly on sapphire (solid line). 
Table 1. Interplanar distances (d) obtained by XRD and SAD from the InN film grown directly on sapphire. The literature $d$ values of $\mathrm{InN}$ are also presented for comparison.

\begin{tabular}{llll}
\hline $\mathrm{hk} \underline{(\mathrm{h}+\mathrm{k}) 1}$ & $\mathrm{~d}_{\mathrm{SAD}}(\AA)$ & $\mathrm{d}_{\mathrm{XRD}}(\AA)$ & $\begin{array}{l}\mathrm{d}_{\mathrm{InN}}(\AA)^{\mathrm{a})} \\
\text { literature }\end{array}$ \\
\hline $10 \underline{10} 0$ & 3.06 & & 3.06 \\
0002 & 2.87 & 2.85 & 2.85 \\
$01 \underline{1} 1 \underline{1}$ & 2.70 & 2.70 & 2.69 \\
$01 \underline{\underline{1}} 2$ & 2.09 & & 2.084 \\
$11 \underline{\underline{2}} 0$ & 1.77 & 1.77 & 1.766 \\
$10 \underline{1} 3$ & 1.61 & & 1.613 \\
$02 \underline{2} 0$ & & & 1.53 \\
$11 \underline{\underline{2}} 2$ & 1.5 & & 1.50 \\
$11 \underline{\underline{2}} 6$ & & 1.47 & 1.48 \\
0004 & & 1.424 & 1.427 \\
$02 \underline{2} 2$ & 1.35 & 1.36 & 1.35 \\
\hline
\end{tabular}

a) JCPDS file \# 79-2498 

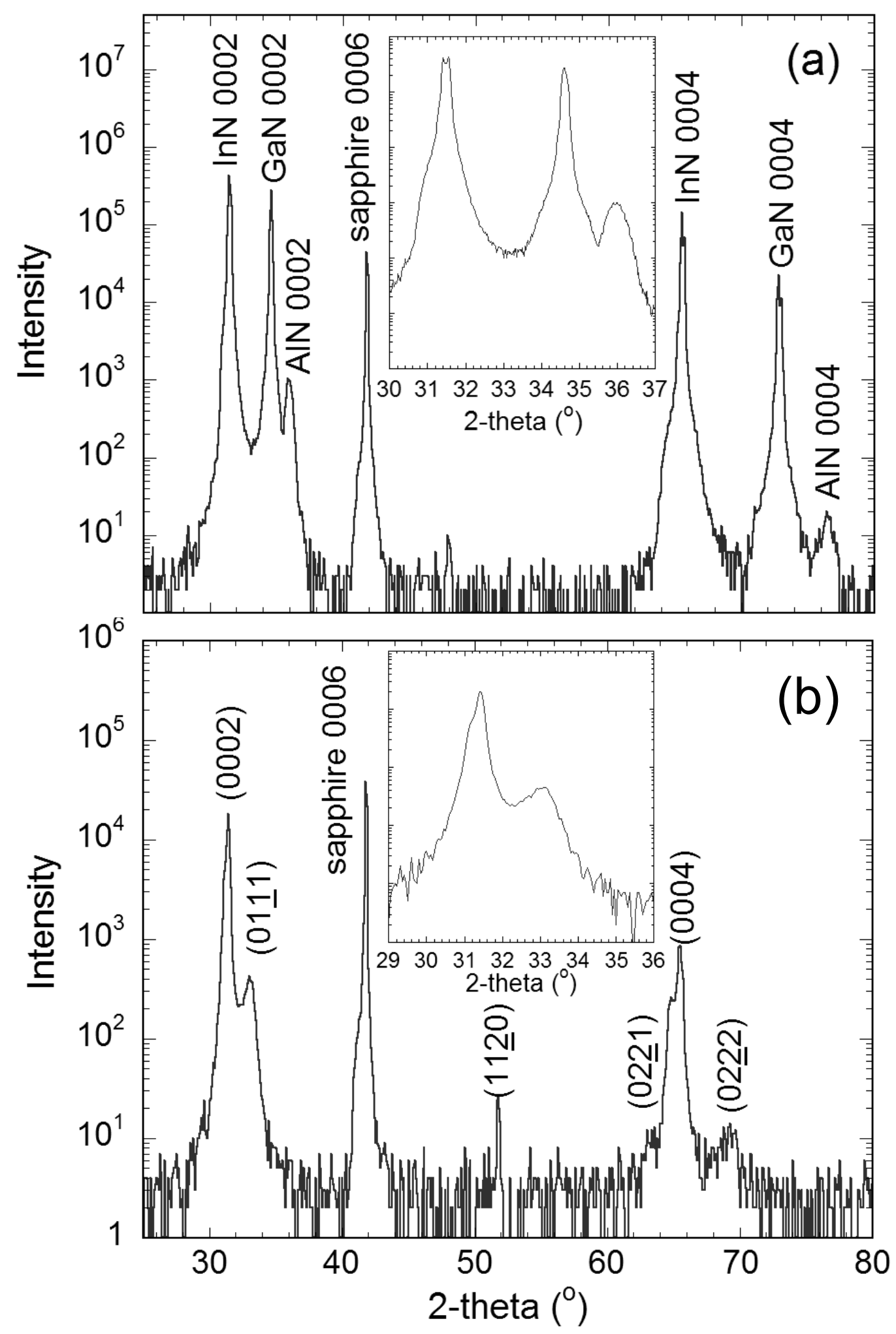

Figure 1 

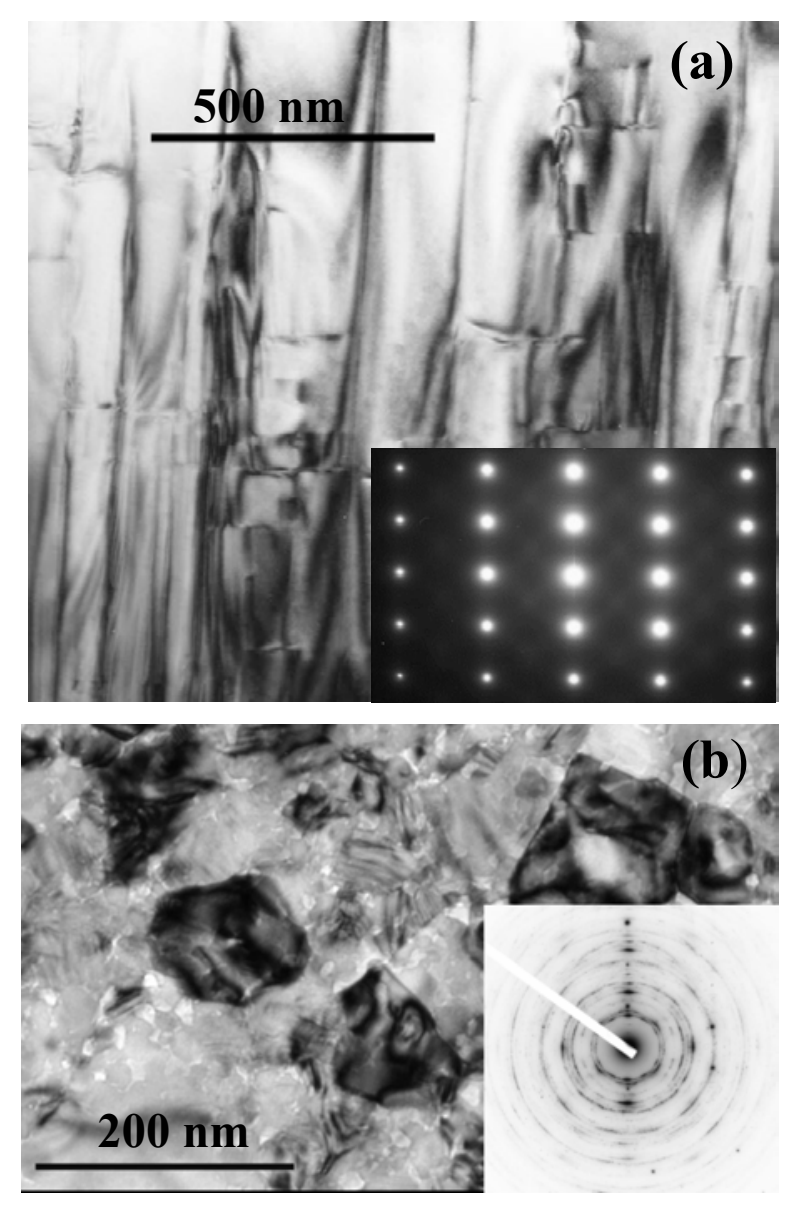

Fig. 2 


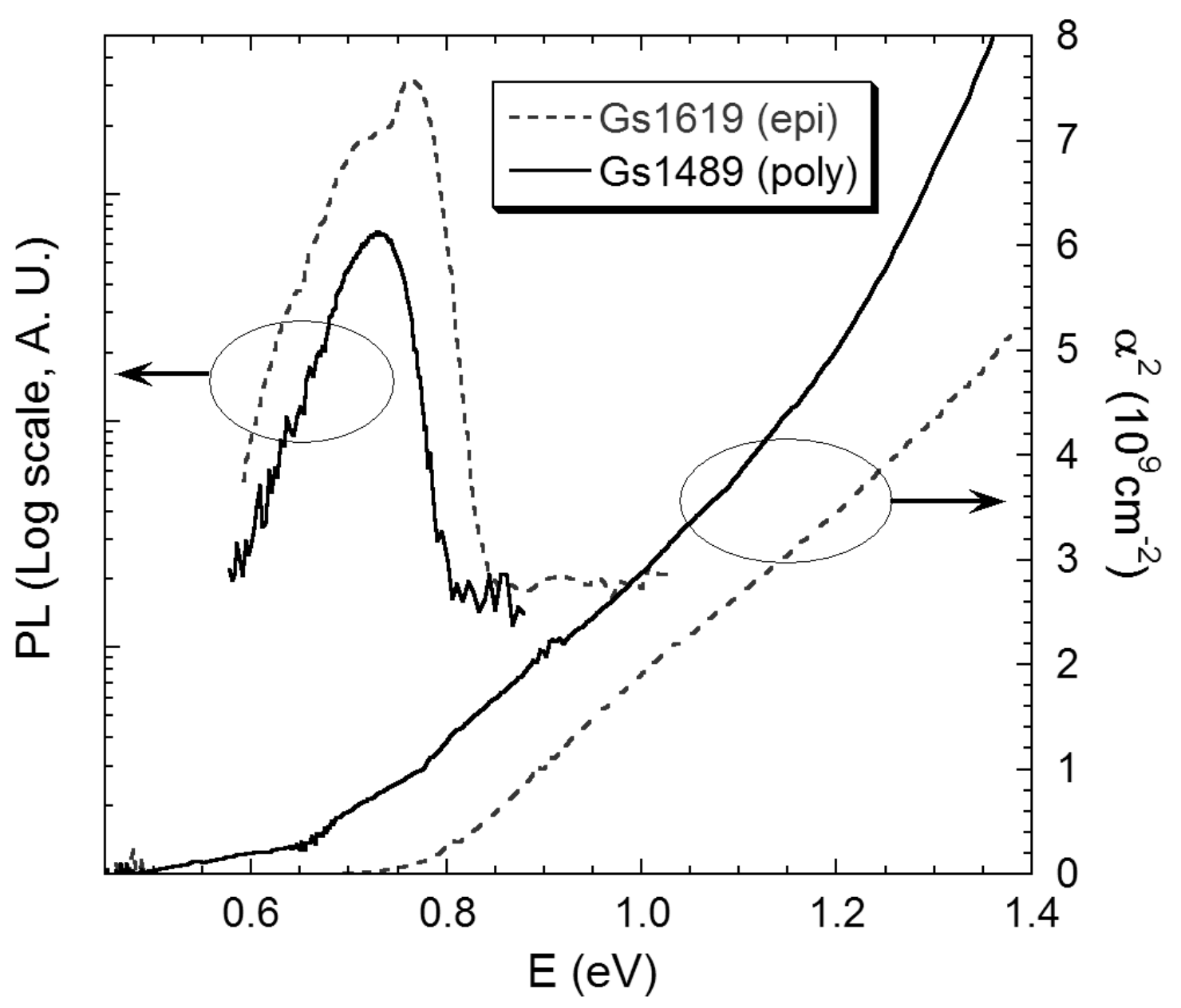

Fig. 3 